\title{
Desempenho acústico de laje com vigota e lajota: isolamento ao som aéreo e de impacto
}

Acoustic performance of $t$-beam and hollow-block slabs: airborne and impact noise insulation

\begin{tabular}{|c|}
\hline $\begin{array}{l}\text { Maria Fernanda Oliveira (D) } \\
\text { Rafael Ferreira Heissler (D) } \\
\text { Felipe Schneider de Lima (D) } \\
\text { Fernanda Pacheco } \\
\text { Bernardo Fonseca Tutikian (D) }\end{array}$ \\
\hline $\begin{array}{l}\text { Resumo } \\
\text { onsiderando o uso expressivo no cenário nacional de lajes pré- } \\
\text { fabricadas de concreto com elementos inertes de preenchimento do } \\
\text { tipo cerâmico, este trabalho contém a avaliação do comportamento do } \\
\text { sistema frente a ruídos aéreos e de impacto, em diferentes } \\
\text { composições. Foram realizados ensaios em câmara reverberante vertical, conforme } \\
\text { a ISO 10140-5:2010. As variações empregadas nos sistemas foram contrapiso, } \\
\text { camada resiliente, revestimentos de laminado de madeira e porcelanato, reboco de } \\
\text { argamassa e forro suspenso. Os resultados evidenciaram que o desempenho } \\
\text { acústico ao som de impacto da laje pré-fabricada de concreto com lajota cerâmica } \\
\text { é afetado pelo efeito da ressonância nas cavidades das lajotas e que a adição de } \\
\text { contrapiso pode compensar o menor isolamento acústico em comparação à laje de } \\
\text { concreto maciço, em razão do consequente aumento na massa do sistema. Os } \\
\text { resultados também mostraram que, para o isolamento ao ruído aéreo, não existem } \\
\text { diferenças significativas entre os revestimentos de porcelanato e piso laminado e } \\
\text { que a instalação do forro compensou a reduzida perda de transmissão sonora da } \\
\text { laje vigota/lajota em relação à laje de concreto maciço. }\end{array}$ \\
\hline
\end{tabular}

${ }^{1}$ Maria Fernanda Oliveira ${ }^{1}$ Unisinos São Leopoldo RS - Brasil

\section{${ }^{2}$ Rafael Ferreira Heissler ${ }^{2}$ Unisinos \\ São Leopoldo RS - Brasil}

${ }^{3}$ Felipe Schneider de Lima
${ }^{3}$ Construrohr
Bom Princípio - RS - Brasil

${ }^{4}$ Fernanda Pacheco ${ }^{4}$ Unisinos

São Leopoldo RS - Brasil

\section{${ }^{5}$ Bernardo Fonseca Tutikian 5 Unisinos São Leopoldo RS - Brasil}

Recebido em 03/03/20 Aceito em 13/11/20
Palavras-chave: Desempenho acústico. Laje pré-fabricada. Ruído de impacto. Ruído aéreo. Sistemas de piso.

\section{Abstract}

Considering the widespread use of T-beam/hollow-block slabs in Brazil, this study examines the behaviour of that system under the effect of airborne and impact noises, in different compositions. Tests were carried out at a specific test facility, according to ISO 10140-5: 2010. The variations used in the systems were: subfloor, resilient layer, wood laminate and porcelain tile floorings, mortar plaster and suspended ceilings. The results showed that the acoustic performance of the T-beam/hollow-block slab is affected by the effect of resonance in the blocks' cavities and that the addition of a subfloor can compensate the acoustic fragility when compared with the concrete slab, because of the consequent increase in the system's mass. The results also show that, for the airborne sound insulation, there are no significant differences between the porcelain tiles and the wood laminate flooring, and that the installation of the suspended ceiling made up for the small sound transmission loss of the T-beam/hollow-block slab in relation to the concrete slab.

Keywords: Acoustic performance. Prefabricated concrete slabs. Impact noise. Airborne sound. Floor systems. 


\section{Introdução}

Os sistemas de piso constituem um limite horizontal de separação entre unidades habitacionais em edifícios, sendo seu desempenho acústico avaliado tanto para o ruído aéreo como para o ruído de impacto. Em alguns sistemas de pisos, considerados não homogêneos, como o caso do sistema de laje pré-fabricada executada em vigota e lajota, o meio de propagação se torna um pouco mais complexo quando comparado a um sistema homogêneo composto por laje de concreto maciço, muitas vezes em função das características ortotrópicas do meio de transmissão (OLIVEIRA; PATRICIO, 2017). Ensaios em laboratório são fundamentais para o entendimento das características desse tipo de sistema construtivo para a adequada previsão de isolamento acústico, pois os métodos usuais de estimativa de redução sonora são mais adequados para sistemas pesados homogêneos (CANIATO et al., 2017).

A composição, a espessura e o tipo de instalação podem caracterizar o sistema de piso como homogêneo, devido ao aumento da espessura total e dos vínculos rígidos, ou heterogêneo, devido à transmissão predominante pelas vigas de concreto colocadas em uma única direção (HOPKINS, 2014; HOPKINS; TURNER, 2005).

$\mathrm{Na}$ laje pré-fabricada do tipo vigota e lajota, a primeira é um elemento estrutural pré-fabricado, composto por concreto e armadura, e a segunda é um elemento de enchimento, sem função estrutural, a ser disposto entre as vigotas, capaz de resistir às solicitações durante as fases de trabalho de montagem e concretagem e suportar o concreto de capeamento do sistema construtivo (ABNT, 2016). Assim, a laje pré-fabricada do tipo vigota e lajota apresenta maior transmissão acústica nos espaços das lajotas, uma vez que permite a reverberação interna do som, com a amplificação das ondas sonoras em determinadas faixas de frequência (HOPKINS, 2012).

A perda de transmissão frente ao ruído aéreo e ao ruído de impacto apresenta diferenças e as soluções construtivas nem sempre atendem simultaneamente esses dois requisitos. O ruído aéreo é originado a partir de sucessivas ondas de pressão ou vibrações que são geradas pela fala ou sistemas de áudio e que são transmitidas pelo ar. Para esses casos, o aumento na massa dos sistemas construtivos dificulta a transmissão das vibrações e, consequentemente, reduz a transmissão sonora para a superfície oposta (BIES; HANSEN, 2017). A estimativa teórica do isolamento acústico do ruído aéreo em sistemas de pisos segue o mesmo princípio da transmissão do ruído aéreo em sistemas de vedações verticais, e podem ser adotados os princípios da Lei da Massa e dos sistemas massa-mola-massa. Porém, a composição dos sistemas de piso envolve diferentes camadas de materiais e, em alguns casos, conta ainda com forros suspensos que têm diversas possibilidades de montagem. Essa situação caracterizada pela diversidade de combinações evidencia a complexidade da aplicação de modelos teóricos (HASSAN, 2009).

O ruído de impacto é caracterizado como a ação de um choque exercido sobre um elemento de compartimentação que pode se propagar através dos materiais e dos elementos construtivos vinculados a um sistema de piso, com a capacidade de difundir a energia sonora em uma ampla faixa de frequência que resulta do movimento vibratório conduzido por uma excitação localizada (HOPKINS, 2012; PATRÍCIO, 2018).

Quanto maior for a massa da superfície em análise, menor será a probabilidade de vibrar e, portanto, de transmitir som; por sua vez, quanto mais rígida e contínua for uma estrutura, menos energia sonora é transmitida através dela (HASSAN, 2009; HOPKINS, 2012). Entretanto, embora a massa de um elemento seja de grande importância para atenuar o ruído aéreo, ela desempenha um papel secundário no isolamento do ruído de impacto, visto que a energia de vibração é maior e é aplicada diretamente à construção, com pouca perda de energia (BIES; HANSEN, 2017; PARK; YOON; CHO, 2020).

Na composição do sistema com vigotas e lajotas, os elementos de enchimento são normalmente compostos por material cerâmico ou por poliestireno expandido (EPS) e, posteriormente, são cobertos por uma camada de concreto, o que garante a distribuição dos esforços atuantes, aumenta a resistência à flexão do elemento, bem como assegura o nivelamento do piso (SEMPRINI et al., 2010). Esse enchimento, além de substituir parte do elemento tracionado da laje, tem a função de sustentar a capa de concreto fresco até seu endurecimento.

A heterogeneidade do sistema vigota/lajota causa diferenças significativas nos mecanismos de transmissão pelos flancos, sendo esse um dos principais fatores que limitam o isolamento acústico nos sistemas com lajes pré-moldadas do tipo vigota/lajota (SCHIAVI; TARIZZO; ASTOLFI, 2010; SEMPRINI et al., 2010).

Para Schiavi, Tarizzo e Astolfi (2010), a radiação acústica em estruturas compostas por vigotas de concreto e lajotas cerâmicas decorre da não difusividade do campo vibracional, da presença de descontinuidades

244 Oliveira, M. F.; Heissler, R. F.; Lima, F. S. de; Pacheco, F.; Tutikian, B. F. 
estruturais dos sistemas ortotrópicos e da complexidade da estrutura frente à irradiação sonora de uma região irregular, como uma laje composta por dois tipos diferentes de materiais. Para os autores, uma laje composta por vigota de concreto e lajota cerâmica pode ser considerada como uma placa rígida com nervuras de concreto rigidamente acoplada a lajotas cerâmicas vazadas, que cooperam na distribuição da carga estática. A resposta acústica à excitação de impacto neste tipo de laje, devido a ressonâncias e alta eficiência de radiação de elementos, especialmente em altas frequências, difere consideravelmente da tendência do espectro de uma laje de concreto monolítica e homogênea, que é considerada uma estrutura rígida.

Para melhorar o isolamento acústico do tipo de laje composto por vigota e lajota, utiliza-se o piso flutuante a fim de nivelar e regularizar o sistema (SCHIAVI, 2018). O piso flutuante é um sistema construtivo composto por um elemento rígido, como um contrapiso ou placa rígida, sobre um material resiliente também denominado "camada elástica", que o desvincula dos elementos estruturais e de vedação da edificação (CANIATO et al., 2017; HOPKINS, 2012; YOO; JEON, 2014). Apesar de sua comprovada eficiência na redução dos sons de impacto, as análises que envolvem o sistema construtivo completo são determinantes para a escolha de soluções adequadas (NUNES; ZINI; PAGNUSSAT, 2014).

O isolamento do ruído de impacto de uma laje é apontado por meio do nível de pressão sonora transmitido, resultando na relação de que quanto maior for o nível indicado, menor será o seu isolamento. Para melhorar o desempenho acústico de uma laje estrutural, além do piso flutuante, podem ser adotados materiais resilientes de revestimento, forros suspensos, contrapiso flutuante ou, até mesmo, a descontinuidade das estruturas (HASSAN, 2009; HOPKINS, 2012; PATRÍCIO, 2018).

Os revestimentos de piso têm função relevante para o controle da transmissão do ruído de impacto, sendo que existem dois sistemas principais para sua atenuação: os revestimentos flexíveis de piso e os sistemas de piso flutuante (BORGES et al., 2018; SCHIAVI, 2018). O primeiro grupo pode ser exemplificado por carpetes ou materiais de base polimérica. Nesse caso, a redução do ruído de impacto é consequência do aumento do tempo de impacto, devido às características do material elástico (PATRÍCIO, 2018). Dessa forma, o aumento do tempo de impacto está relacionado às características elásticas do revestimento do piso, que reduzirá o espectro de excitação a ser irradiado pela laje estrutural. Por sua vez, os sistemas de piso flutuante, como já citado, empregam um material rígido e pesado em cima da uma camada resiliente.

Para Semprini e Barbaresi (2013), o desempenho acústico ao ruído de impacto da laje de vigota e lajota são muito diferentes do analisado para uma laje de concreto monolítica e homogênea, uma vez que no sistema construtivo executado em vigota e lajota há altos níveis de radiação acústica, que ocorrem em altas frequências, principalmente devido a ressonâncias de cavidades das peças vazadas. Os autores concluíram que existe maior resposta vibracional do sistema a partir das bandas de médias e altas frequências, quando comparado a uma laje de concreto maciço. O aumento nas bandas de altas frequências resulta em maiores valores ponderados, como consequência do maior peso para os sons mais agudos no cálculo do valor ponderado.

Oliveira e Patrício (2017) apresentam resultados de simulação em modelos computacionais por análise de elementos finitos da resposta vibracional de lajes não homogêneas submetidas ao ensaio de nível de pressão sonora de impacto. $\mathrm{O}$ estudo apresenta modelos de lajes simulados com capa de $5 \mathrm{~cm}$ de concreto. Os autores afirmam que, na comparação entre os resultados dos sistemas de laje maciça, laje de vigota e lajota, laje nervurada e laje nervurada com cubetas de EPS, a maior rigidez estrutural da laje nervurada não representou maior eficiência na redução da irradiação das vibrações. Ainda, o preenchimento da laje nervurada com cubetas de EPS não alterou significativamente o comportamento da laje do tipo nervurada sem preenchimento das cubetas. Nesse estudo, o modelo computacional da laje do tipo vigota/lajota apresentou maior pico vibracional nas altas frequências, o que representa menor capacidade de isolamento aos sons de impacto.

O sistema de forro suspenso não é considerado tão eficiente para o isolamento ao ruído de impacto quanto o tratamento do sistema construtivo na própria superfície em que é gerada a excitação mecânica. No entanto, a utilização dessa técnica se torna uma das poucas alternativas quando não é possível realizar algum tratamento no ambiente emissor do ruído (MOSHARROF et al., 2011; TOMITAKA; NOJIMA; MASUDA, 2016). Nesses casos, como em edifícios de múltiplos pavimentos, a instalação de um forro pode minimizar falhas no isolamento acústico, que costumam estar relacionadas aos fatores de desconforto dos usuários (KIM; JEON, 2014).

Nesse contexto, o objetivo deste artigo foi caracterizar o isolamento acústico ao som aéreo e de impacto em laboratório de composições de pisos com laje pré-fabricada do tipo vigota e lajota cerâmica. Também foi 
avaliada a presença de forro de gesso, manta acústica, contrapiso de argamassa, pisos do tipo laminado e porcelanato e reboco de argamassa, como elementos que compunham o sistema construtivo.

\section{Materiais e métodos}

\section{Materiais}

O elemento estrutural utilizado neste estudo foi uma laje pré-fabricada unidirecional tipo LP13, com intereixo - isto é, distância entre eixos centroidais de duas vigotas paralelas adjacentes - de $47 \mathrm{~cm}$, composta por vigotas de concreto protendido, lajotas cerâmicas Hc 8/37/20 (8cm de altura, $37 \mathrm{~cm}$ de largura e $20 \mathrm{~cm}$ de comprimento) e capa de concreto de $5 \mathrm{~cm}$ de espessura com resistência característica à compressão $\left(f_{c k}\right)$ de $25 \mathrm{MPa}$. A capa de concreto foi executada com concreto usinado classe $\mathrm{C} 25$, com abatimento de tronco de cone classe $\mathrm{S} 100$ e densidade de massa aparente de $2400 \mathrm{~kg} / \mathrm{m}$.

Para fins comparativos, também foi ensaiada uma laje de concreto maciço. Os materiais utilizados nas composições dos sistemas de piso estão descritos a seguir, com as respectivas nomenclaturas adotadas neste trabalho:

(a) VL - laje pré-fabricada unidirecional tipo LP13, com intereixo de $47 \mathrm{~cm}$, composta por vigotas de concreto protendido, lajotas cerâmicas Hc 8/37/20 e capa de concreto de 50mm de espessura;

(b) F - forro de gesso acartonado com placas do tipo standard, na espessura de 12,5mm, com distância entre o forro e a face inferior da laje (plenum) de 150mm;

(c) C - contrapiso de 40mm de espessura confeccionado com argamassa cimentícia industrializada marca Votoran peso específico de $1800 \mathrm{~kg} / \mathrm{m}$;

(d) P - piso de porcelanato cerâmico, marca Delta ${ }^{\circledR}$ Porcelanato, modelo Formato Prático, dimensões de $54 \mathrm{~cm} \times 54 \mathrm{~cm}$ com espessura de $8,9 \mathrm{~mm}$;

(e) L - piso laminado de madeira, marca Eucafloor, modelo Prime, classe de abrasão AC3, com dimensões de $135,7 \mathrm{~cm} \times 19,7 \mathrm{~cm}$ e espessura de $7 \mathrm{~mm}$ sobre manta de proteção mecânica de polietileno expandido com espessura de $2 \mathrm{~mm}$;

(f) R - reboco com argamassa cimentícia industrializada na espessura de $20 \mathrm{~mm}$; e

(g) CM - laje de concreto maciço usinado C30, com espessura de $120 \mathrm{~mm}$, abatimento de tronco de cone classe S150, armadura em aço CA-50 com cobrimento de $20 \mathrm{~mm}$ e densidade de massa aparente de $2500 \mathrm{~kg} / \mathrm{m}$.

A caracterização do isolamento ao ruído aéreo e ao som de impacto foi realizada em laboratório para oito configurações de sistemas de piso com laje pré-fabricada de concreto e para a laje de concreto maciço, conforme mostra o Quadro 1.

As amostras foram confeccionadas em um suporte metálico com dimensões internas de 4,01m $\times 4,11 \mathrm{~m}$ com a distribuição das vigotas no sentido da menor dimensão da estrutura metálica (Figura 1a). As etapas seguintes incluíram a confecção do capeamento (Figura 1b), a instalação do forro suspenso (Figura 1c) e a instalação dos revestimentos de piso (Figura 1d).

Os ensaios foram realizados em laboratório acreditado pela Coordenação Geral de Acreditação do Inmetro, em câmaras reverberantes construídas e validadas conforme a ISO 10140-5 (INTERNATIONAL..., 2010d) e com equipamentos calibrados.

O uso do suporte metálico permitiu o içamento dos sistemas para a câmara de ensaio (Figura 2a). A amostra foi instalada sobre a câmara de recepção e, posteriormente, a câmara de emissão foi posicionada sobreposta (Figura 2b). Para os ensaios com os sistemas de pisos compostos por laje pré-fabricada de concreto, não ocorreu movimentação entre as configurações, mantendo a condição de vínculo em todas as situações da pesquisa. 
Quadro 1 - Caracterização das amostras

\begin{tabular}{|c|c|c|}
\hline Sigla da amostra & Descrição & Esquema construtivo \\
\hline VL & $\begin{array}{l}\text { Laje pré-fabricada composta } \\
\text { por vigotas de concreto } \\
\text { protendido, lajotas cerâmicas } \\
\text { e capa de concreto. }\end{array}$ & \\
\hline VLF & $\begin{array}{l}\text { VL com a instalação de forro } \\
\text { de gesso acartonado com } \\
\text { plenum de } 150 \mathrm{~mm} \text {. }\end{array}$ & \\
\hline VLFC & $\begin{array}{l}\text { VLF com contrapiso de } \\
40 \mathrm{~mm} \text { de espessura. }\end{array}$ & \\
\hline VLFCP & $\begin{array}{l}\text { VLFC com revestimento de } \\
\text { porcelanato cerâmico. }\end{array}$ & \\
\hline VLFCL & $\begin{array}{l}\text { VLFC com revestimento de } \\
\text { piso laminado sobre manta } \\
2 \mathrm{~mm} .\end{array}$ & \\
\hline VLCL & $\begin{array}{l}\text { VLFCL sem a instalação de } \\
\text { forro de gesso acartonado } \\
\text { com plenum de } 150 \mathrm{~mm} \text {. }\end{array}$ & \\
\hline VLCP & $\begin{array}{l}\text { VLFCP sem a instalação de } \\
\text { forro de gesso acartonado } \\
\text { com plenum de } 150 \mathrm{~mm} \text {. }\end{array}$ & \\
\hline VLCPR & $\begin{array}{l}\text { VLCP com a face inferior } \\
\text { com reboco } 20 \mathrm{~mm}\end{array}$ & \\
\hline CM & $\begin{array}{l}\text { Laje de concreto maciço com } \\
\text { espessura de } 120 \mathrm{~mm} \text {. }\end{array}$ & \\
\hline
\end{tabular}


Figura 1 - Etapas de instalação das amostras: (a) instalação das vigotas e lajotas; (b) capeamento; (c) tratamento das frestas entre as placas do forro; e (d) instalação de revestimentos

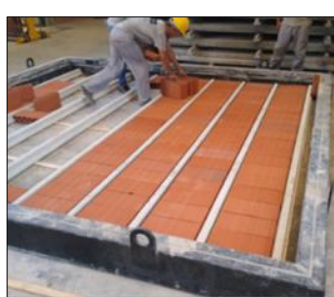

(a)

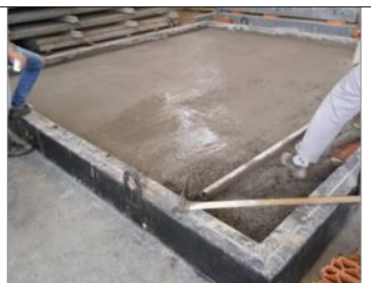

(b)

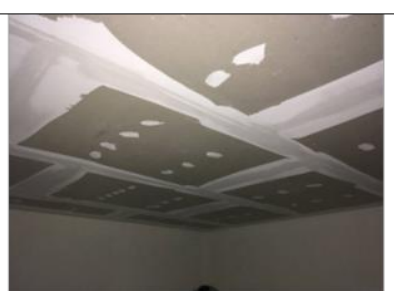

(c)

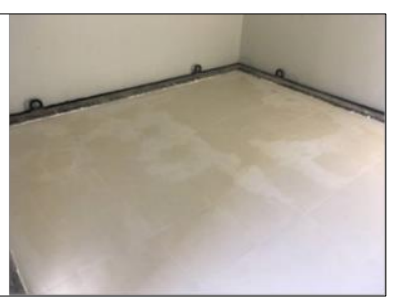

(d)

Figura 2 - Instalação da amostra e da câmara de emissão para o ensaio de ruído de impacto: (a) içamento da amostra de ensaio; e (b) sobreposição da câmara de emissão

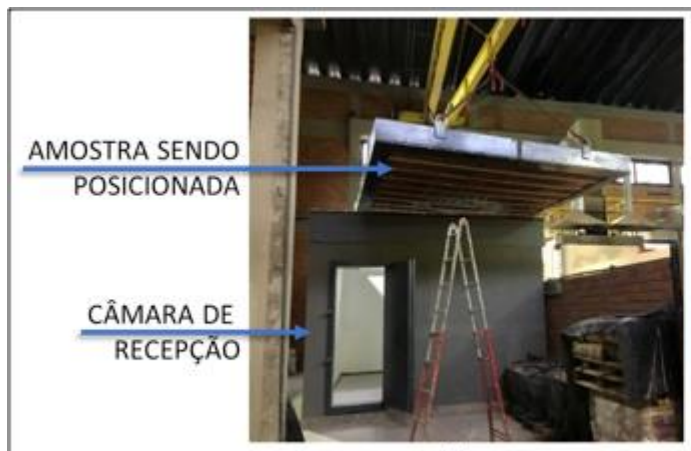

(a)

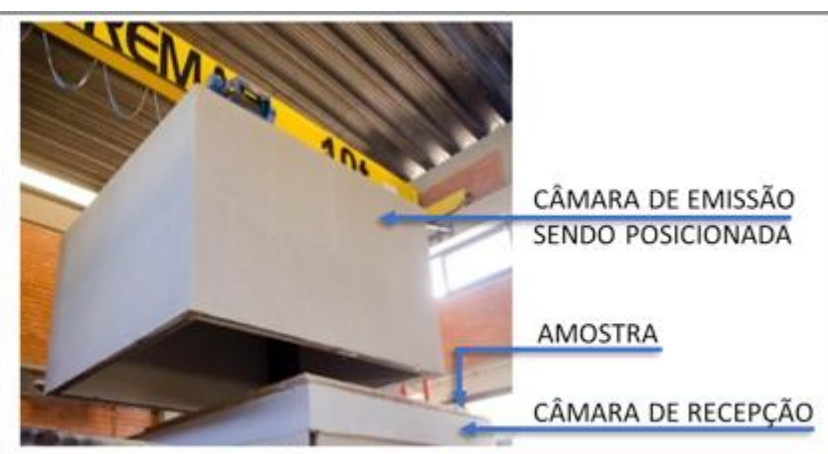

(b)

O isolamento acústico ao som aéreo, expresso pelo índice de redução sonora $(\mathrm{R})$ foi determinado com base em ensaios conforme os procedimentos das normas ISO 10140-2 (INTERNATIONAL..., 2010a) e ISO 10140-4 (INTERNATIONAL..., 2010c). A determinação do índice de redução sonora ponderado $R_{w}$ foi realizada conforme a ISO 717-1 (INTERNATIONAL..., 2013a). Os ensaios para determinar os níveis de pressão sonora de impacto normalizado $\left(L_{n}\right)$ seguiram os procedimentos das normas ISO 10140-3 (INTERNATIONAL..., 2010b) e ISO 10140-4 (INTERNATIONAL..., 2010c). A determinação do número único do nível de pressão sonora de impacto normalizado ponderado $\left(L_{n, w}\right)$ seguiu os procedimentos da ISO 717-2 (INTERNATIONAL..., 2013b). Todas as medições foram feitas em bandas de terço de oitava entre 100 e $5.000 \mathrm{~Hz}$.

Os equipamentos utilizados para as medições acústicas são do fabricante Acoem $01 \mathrm{~dB}$, sendo eles: fonte sonora dodecaédrica onidirecional KIT LS02, amplificador de potência KIT LS02, analisador sonoro 01dB FUSION, microfone G.R.A.S. com pré-amplificador FUSION e calibrador sonoro Cal 21.

\section{Resultados}

Os resultados são apresentados por bandas de terço de oitava, para o isolamento do som aéreo e para os níveis de pressão sonora de impacto normalizado, seguidas pela comparação dos valores ponderados de $R_{w}$ e $L_{n, w}$.

A Figura 3 apresenta o gráfico com os resultados de perda de transmissão sonora por bandas de terço de oitava das oito composições ensaiadas.

Comparando os resultados da laje vigota/lajota (VL) com os resultados da laje de concreto maciço (CM), pode-se perceber que, a partir da banda de $400 \mathrm{~Hz}$, o efeito da ressonância nas cavidades das lajotas afeta o desempenho acústico do sistema de piso, com diferenças que chegam a $21 \mathrm{~dB}$ na banda de $2 \mathrm{kHz}$. No entanto, a adição de contrapiso aumenta a capacidade de isolamento dos sons aéreos da laje VL em comparação à laje de CM, com o aumento da espessura do elemento monolítico formado nas camadas superiores com o capeamento e o contrapiso, com espessura adicionada de $9 \mathrm{~cm}$ e o consequente aumento da massa do sistema.

Os resultados também evidenciam que, para fins de isolamento do ruído aéreo, não há diferenças significativas entre os revestimentos de porcelanato e o piso laminado (amostras VLCL e VLCP). 
A instalação do forro suspenso compensou a reduzida perda de transmissão sonora da laje VL em relação à laje de CM, com aumento do isolamento acústico a partir da banda de $250 \mathrm{~Hz}$.

Os valores apresentados na Figura 3 estão transcritos na Tabela 1 para uma melhor comparação dos dados levantados.

Com relação ao nível de pressão sonora de impacto, nota-se que a adição progressiva das camadas resultou em menores valores de $L_{n, w}$ (Figura 4).

A laje sem revestimento e sem forro, denominada "VL", apresentou uma curva ascendente até a banda de $2 \mathrm{kHz}$, com maior componente tonal nos sons agudos comparado às demais composições e consequente redução no isolamento acústico nessas bandas de frequências. É possível afirmar que a laje do tipo vigota/lajota, quando comparada a uma laje de $\mathrm{CM}$, apresenta pior eficiência para o isolamento nas bandas de altas frequências, em decorrência do efeito de ressonância no interior dos vazios dos elementos cerâmicos (OLIVEIRA; PATRICIO, 2017; PATRÍCIO, 2001; SEMPRINI et al., 2010).

Figura 3 - Resultados por bandas de frequências do isolamento do som aéreo

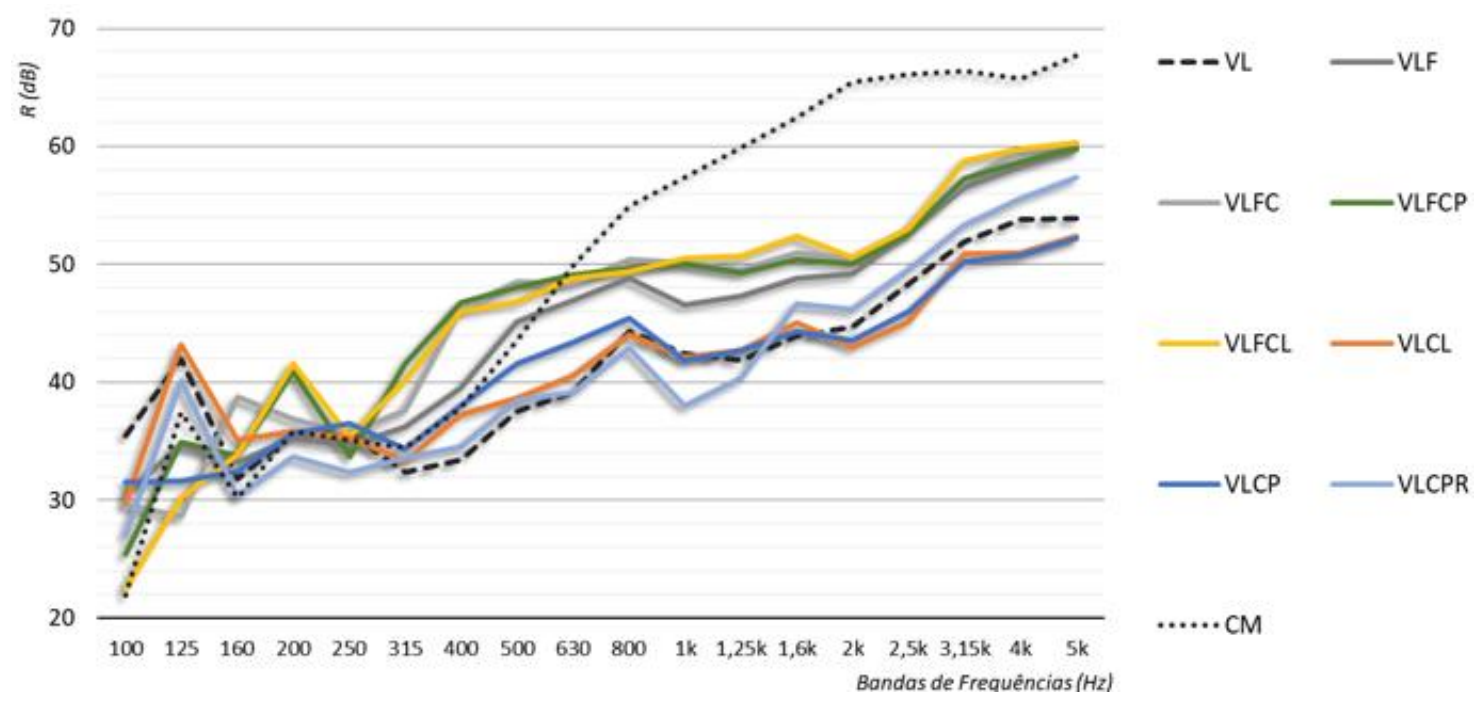

Tabela 1 - Resultados por bandas de frequências do isolamento ao som aéreo (em $\mathrm{dB})$.

\begin{tabular}{c|c|c|c|c|c|c|c|c|c}
\hline $\begin{array}{c}\text { Bandas } \\
\text { centrais } \\
(\mathbf{H z})\end{array}$ & $\mathbf{V L}$ & $\mathbf{V L F}$ & $\mathbf{V L F C}$ & VLFCP & VLFCL & VLCL & VLCP & VLCPR & CM \\
\hline 100 & 35,4 & 30,5 & 29,6 & 25,5 & 22,6 & 29,8 & 31,5 & 27,0 & 21,93 \\
125 & 42,0 & 34,7 & 28,6 & 34,9 & 30,2 & 43,2 & 31,6 & 40,1 & 37,46 \\
160 & 31,8 & 33,2 & 38,8 & 33,8 & 33,9 & 35,1 & 32,4 & 30,3 & 30,18 \\
200 & 35,6 & 35,3 & 36,9 & 41,0 & 41,6 & 35,9 & 35,6 & 33,7 & 35,82 \\
250 & 35,6 & 34,7 & 35,7 & 33,7 & 35,4 & 35,3 & 36,5 & 32,4 & 35,09 \\
315 & 32,4 & 36,2 & 37,7 & 41,6 & 40,2 & 33,3 & 34,4 & 33,6 & 34,46 \\
400 & 33,4 & 39,5 & 46,2 & 46,7 & 46,0 & 37,2 & 38,0 & 34,6 & 37,84 \\
500 & 37,5 & 45,2 & 48,6 & 48,1 & 46,9 & 38,6 & 41,6 & 38,5 & 43,57 \\
630 & 39,1 & 47,0 & 48,4 & 49,1 & 48,8 & 40,6 & 43,4 & 39,1 & 49,81 \\
800 & 44,3 & 48,9 & 50,4 & 49,7 & 49,4 & 44,0 & 45,4 & 42,9 & 54,92 \\
$1 \mathrm{k}$ & 42,4 & 46,5 & 50,1 & 50,0 & 50,5 & 42,2 & 41,9 & 38,0 & 57,34 \\
$1,25 \mathrm{k}$ & 41,9 & 47,3 & 49,6 & 49,2 & 50,7 & 42,7 & 42,7 & 40,3 & 59,92 \\
$1,6 \mathrm{k}$ & 43,9 & 48,8 & 51,0 & 50,5 & 52,4 & 45,0 & 44,3 & 46,6 & 62,46 \\
$2 \mathrm{k}$ & 44,7 & 49,2 & 50,5 & 50,0 & 50,6 & 43,0 & 43,6 & 46,2 & 65,45 \\
$2,5 \mathrm{k}$ & 48,4 & 52,7 & 52,8 & 52,5 & 53,1 & 45,2 & 46,0 & 49,6 & 66,12 \\
$3,15 \mathrm{k}$ & 51,9 & 56,5 & 57,0 & 57,2 & 58,8 & 50,9 & 50,2 & 53,3 & 66,42 \\
$4 \mathrm{k}$ & 53,8 & 58,3 & 59,6 & 58,7 & 59,8 & 51,0 & 50,8 & 55,6 & 65,75 \\
$5 \mathrm{k}$ & 53,9 & 59,7 & 60,3 & 59,9 & 60,3 & 52,4 & 52,2 & 57,4 & 67,71 \\
\hline
\end{tabular}


Figura 4 - Resultados por bandas de frequências do nível de ruído de impacto

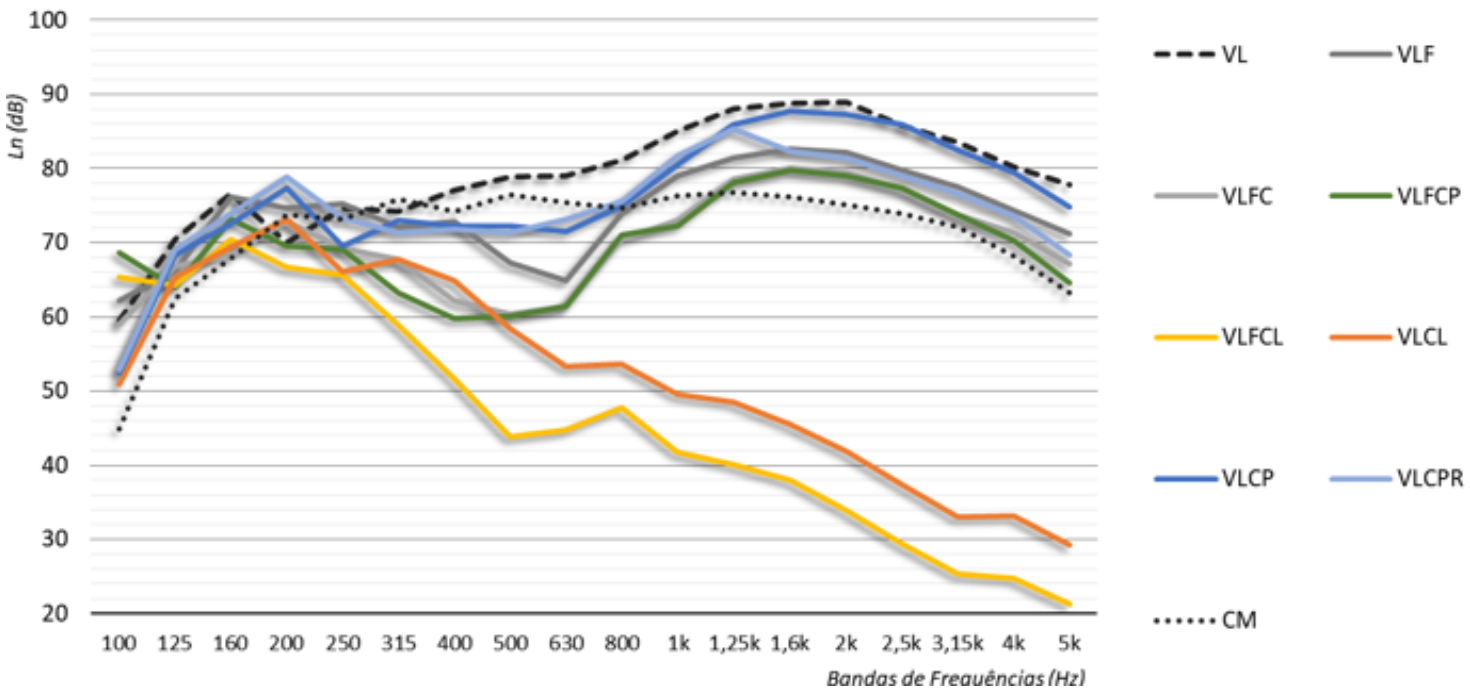

A adição de $4 \mathrm{~cm}$ de contrapiso e revestimento de porcelanato, amostra VLCP, acarretou redução dos níveis sonoros medidos a partir da banda de $400 \mathrm{~Hz}$, comparando-se com a amostra VL. No entanto, o perfil gráfico manteve a característica de um sistema rígido, com aumento dos níveis sonoros até a banda de $2 \mathrm{kHz}$. A redução dos níveis sonoros sem a alteração do perfil gráfico é característica em situações em que se aumenta a espessura do sistema de piso sem a instalação de algum recurso de amortecimento, conforme indicado por Hassan (2009). Dessa forma, pode-se afirmar que a utilização do contrapiso com espessura de $4 \mathrm{~cm}$ aplicado a uma laje do tipo vigota/lajota pode ser uma alternativa viável para aumentar o isolamento sonoro do sistema construtivo, mas que não reduz de forma eficiente o maior incômodo causado pelos sons agudos.

A amostra VLFCP, composta por contrapiso, forro de gesso e revestimento de porcelanato, apresentou os mesmos valores que a amostra sem revestimento (VLFC). Apesar da importância do revestimento de piso no controle do ruído de impacto, a característica elástica dessas superfícies exerce maior influência em relação ao amortecimento do impacto mecânico. Sendo assim, não ocorre a redução do espectro irradiado pelo elemento estrutural da laje. Por outro lado, as amostras com revestimento em piso laminado apresentaram maior redução dos níveis sonoros, sendo que com a amostra VLFCL, com forro de gesso e piso laminado, verifica-se o perfil gráfico típico de um sistema com amortecimento eficiente, tendo o decaimento dos níveis sonoros a partir da banda de $200 \mathrm{~Hz}$. Esse decaimento é típico em sistemas com piso flutuante, em que a resposta vibracional do sistema é sensivelmente atenuada no ponto de excitação mecânica primária, conforme pode ser constatado também em outros trabalhos (LEE; KIM; LIM, 2014; PARK; YOO; CHO, 2020). É importante ressaltar que, conceitualmente, os sistemas com piso laminado de madeira não podem ser classificados como um piso flutuante, pois não têm massa suficiente da camada superior, nem amortecimento característico na camada elástica devido à manta ser somente de proteção mecânica. Nas amostras VLFCL e VLCL se verifica, portanto, o efeito de um revestimento flutuante.

Os valores apresentados na Figura 4 estão transcritos na Tabela 2 para uma melhor comparação dos dados dispostos.

Os resultados ponderados podem ser analisados no gráfico da Figura 5. Para o ruído aéreo, os valores maiores de $R_{w}$ representam maior isolamento acústico; por sua vez, para o ruído de impacto, os valores menores de $L_{n, w}$ indicam melhores condições de isolamento acústico.

De modo geral, pode-se afirmar que as soluções adotadas para o aumento da eficiência do isolamento do som aéreo não necessariamente apresentam a mesma eficiência para a redução dos sons de impacto. Nesse contexto, pode-se destacar uma exceção apresentada nos valores da amostra VLFCL, composta por forro suspenso, contrapiso e piso laminado. Nessa amostra foi obtido o maior valor e $R_{w}$ e o menor valor de $L_{n, w}$.

Os maiores resultados de $R_{w}$ foram obtidos com as composições com forro suspenso, e os menores valores de $L_{n, w}$ foram influenciados diretamente pela adoção do revestimento de piso laminado com manta resiliente. A instalação do forro suspenso de gesso acartonado resultou em um acréscimo de $5 \mathrm{~dB}$ em $R_{w}$ nos sistemas somente com a laje vigota/lajota e nos sistemas com revestimento em porcelanato cerâmico. Nos sistemas de piso com revestimento em laminado, a diferença de $R_{w}$ foi de $6 \mathrm{~dB}$. 
As maiores diferenças entre os resultados podem ser ainda indicadas para as amostras com forro suspenso. A amostra somente forro suspenso (VLF) resultou na diferença de $5 \mathrm{~dB}$ em relação às amostras VL e VLCPR e de $4 \mathrm{~dB}$ em relação à amostra VLCL. As amostras com forro e contrapiso e/ou piso (VLFC, VLFCP e VLFCL) resultaram na diferença de $7 \mathrm{~dB}$ em relação às amostras VL e VLCPR, de $6 \mathrm{~dB}$ em relação à VLCL e de $5 \mathrm{~dB}$ em relação à VLCP.

A influência da instalação do forro de gesso no ruído de impacto ponderado pode ser verificada na comparação entre os sistemas VLCP $\times$ VLFCP, VL $\times$ VLF e VLCL $\times$ VLFCL, com diferenças de 9, 7 e $3 \mathrm{~dB}$, respectivamente. Evidencia-se, portanto, que o uso do forro suspenso pode atuar na redução dos sons de impacto e que a diferença é maior nas instalações sem revestimento resiliente. Os valores encontrados com os sistemas com laje pré-fabricada vigota/lajota divergem em parte dos valores encontrados em outros estudos que indicam a diferença de, aproximadamente, $10 \mathrm{~dB}$ em sistemas com lajes maciças (TOMITAKA; NOJIMA; MASUDA, 2016) e de $21 \mathrm{~dB}$ em sistema com laje nervurada com cubetas de EPS (BET et al., 2019).

Tabela 2 - Resultados por bandas de frequências do nível de ruído de impacto (em dB)

\begin{tabular}{c|c|c|c|c|c|c|c|c|c}
\hline $\begin{array}{c}\text { Bandas } \\
\text { centrais } \\
(\mathbf{H z})\end{array}$ & $\mathbf{V L}$ & $\mathbf{V L F}$ & $\mathbf{V L F C}$ & $\mathbf{V L F C P}$ & $\mathbf{V L F C L}$ & $\mathbf{V L C L}$ & $\mathbf{V L C P}$ & VLCPR & CM \\
\hline 100 & 59,4 & 62,2 & 59,0 & 68,7 & 65,3 & 50,9 & 52,4 & 52,9 & 44,8 \\
125 & 70,5 & 66,2 & 66,8 & 63,9 & 64,3 & 65,4 & 68,4 & 69,1 & 62,5 \\
160 & 76,7 & 76,2 & 68,4 & 73,1 & 70,5 & 69,4 & 72,5 & 74,0 & 67,9 \\
200 & 70,0 & 74,6 & 72,8 & 69,5 & 66,7 & 73,1 & 77,4 & 78,9 & 73,7 \\
250 & 74,6 & 75,3 & 69,2 & 69,0 & 65,6 & 66,1 & 69,6 & 73,3 & 73,2 \\
315 & 74,2 & 72,2 & 67,7 & 63,3 & 58,9 & 67,7 & 73,0 & 71,3 & 75,9 \\
400 & 77,1 & 72,8 & 62,1 & 59,8 & 51,7 & 65,0 & 72,2 & 71,8 & 74,3 \\
500 & 78,8 & 67,3 & 60,4 & 60,1 & 43,9 & 58,4 & 72,3 & 71,3 & 76,5 \\
630 & 79,0 & 64,9 & 61,5 & 61,4 & 44,8 & 53,2 & 71,5 & 73,1 & 75,4 \\
800 & 81,1 & 73,9 & 70,5 & 71,0 & 47,7 & 53,5 & 75,0 & 75,6 & 74,7 \\
$1 \mathrm{k}$ & 85,0 & 79,0 & 73,0 & 72,3 & 41,8 & 49,6 & 80,5 & 81,7 & 76,3 \\
$1,25 \mathrm{k}$ & 88,1 & 81,4 & 78,6 & 78,2 & 40,1 & 48,5 & 85,9 & 85,4 & 76,8 \\
$1,6 \mathrm{k}$ & 88,9 & 82,7 & 80,1 & 79,8 & 38,0 & 45,4 & 87,7 & 82,3 & 76,2 \\
$2 \mathrm{k}$ & 88,9 & 82,2 & 79,5 & 79,0 & 33,9 & 41,9 & 87,3 & 81,5 & 75,1 \\
$2,5 \mathrm{k}$ & 85,8 & 79,7 & 77,3 & 77,3 & 29,4 & 37,3 & 85,9 & 79,1 & 73,9 \\
$3,15 \mathrm{k}$ & 83,5 & 77,5 & 73,8 & 73,7 & 25,4 & 33,0 & 82,5 & 76,6 & 72,1 \\
$4 \mathrm{k}$ & 80,3 & 74,4 & 71,4 & 70,3 & 24,7 & 33,2 & 79,4 & 73,6 & 68,2 \\
$5 \mathrm{k}$ & 77,8 & 71,1 & 67,1 & 64,6 & 21,3 & 29,3 & 74,8 & 68,4 & 63,2 \\
\hline
\end{tabular}

Figura 5 - Resultados ponderados das amostras ensaiadas (em dB)

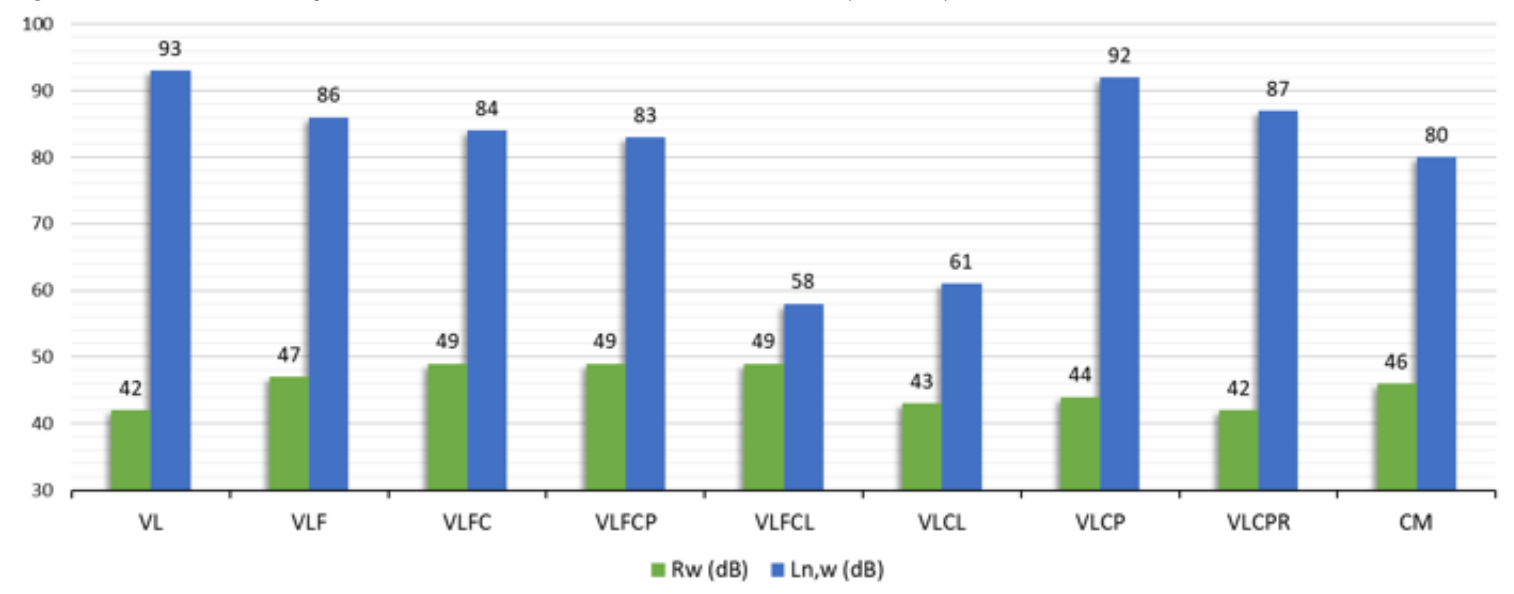


O elevado valor de $L_{n, w}$ de $93 \mathrm{~dB}$ da amostra VL pode ser justificado pela falta de rigidez da estrutura da laje considerada não homogênea e ortotrópica, como já indicado por Hopkins (2012), frente à comparação de uma laje de CM, cuja resposta acústica é mais conhecida dadas as suas características de elemento homogêneo. Os resultados da laje de $\mathrm{CM}$ e da amostra com contrapiso e revestimento de porcelanato (VLCP) podem ser comparados. A amostra VLCP - com $5 \mathrm{~cm}$ de concreto mais $4 \mathrm{~cm}$ de contrapiso e um total de $9 \mathrm{~cm}$ de material rígido com distribuição homogênea em sua área - teve uma diferença de $12 \mathrm{~dB} \mathrm{em}$ relação à laje $\mathrm{CM}$, de $12 \mathrm{~cm}$ de espessura. Essa diferença não se aproxima do indicado por Hassan (2009) que estima em $1 \mathrm{~dB}$ de redução no valor ponderado para cada centímetro de espessura de concreto maciço, pois a diferença de espessura da camada monolítica é de $3 \mathrm{~cm}$. Assim, é possível afirmar que a vigota não é suficiente para fornecer a rigidez necessária para o aumento do isolamento acústico do sistema. Da mesma forma que nos resultados por bandas de frequências, os valores ponderados também evidenciaram a relevante contribuição do amortecimento do revestimento de piso com manta resiliente, com $31 \mathrm{~dB}$ de diferença nos resultados entre VLCP e VLCL, respectivamente, sistemas com revestimento de porcelanato e revestimento laminado.

\section{Conclusão}

Os sistemas de pisos com lajes não homogêneas apresentam maior complexidade para estimar o isolamento acústico. Deve-se considerar, no entanto, que esse tipo de sistema ainda é largamente utilizado em vários países, com custo e carga estrutural reduzidos quando comparados aos sistemas de CM, devendo ser realizados estudos para sua caracterização.

Neste trabalho foram avaliadas diferentes composições de sistemas de pisos com laje do tipo vigota/lajota a partir de ensaios em laboratório. Ao analisar os resultados individuais das composições ensaiadas, percebese que a adição de camadas - como o contrapiso, revestimentos ou o forro suspenso de gesso — contribuiu para melhorar o desempenho acústico da laje vigota/lajota, com a redução do efeito característico de um sistema ortotrópico e da ressonância nas cavidades das lajotas, tanto para os sons aéreos quanto para o ruído de impacto.

A influência do revestimento em sistemas de pisos com lajes não homogêneas é relevante somente para os sons de impacto, estando sua ação diretamente relacionada com a característica elástica da superfície. Para o isolamento do ruído aéreo, não há diferença significativa entre os revestimentos de porcelanato e de piso laminado, considerando a mesma espessura de revestimento.

A instalação do forro de gesso acartonado representou o aumento da capacidade de isolamento dos sons aéreos dos sistemas ensaiados. No entanto, deve-se destacar que as componentes responsáveis pela transmissão por flancos devem ser consideradas nas estimativas para isolamento acústico do edifício como um todo, pois a instalação de um forro suspenso não minimiza as transmissões que ocorrem para os sistemas de vedações verticais.

A laje pré-fabricada com vigotas e lajota, somente com capeamento de $5 \mathrm{~cm}$, revelou menor capacidade de isolamento dos sons de impacto nas altas frequências e um elevado $L_{n, w}$ de $93 \mathrm{~dB}$. Nesse sentido, pode-se afirmar que o isolamento acústico dos sons de impacto em lajes não homogêneas é atribuído principalmente à camada homogênea formada pelo capeamento, sendo desconsiderado, para fins de estimativas de isolamento acústico, o conjunto de vigotas e lajotas.

Pode-se, portanto, indicar que as vantagens do uso de um sistema de piso com laje não homogênea, que visem principalmente a redução de cargas na estrutura, devem ser avaliadas de forma integrada com a estimativa de isolamento acústico requerido para o uso de cada ambiente dos edifícios. Ainda nesse sentido, ressalta-se a necessidade da continuidade e do avanço da pesquisa, com a finalidade de aumentar a contribuição para especificações na etapa de projeto, além de desenvolver novos materiais e sistemas construtivos que atendam critérios de desempenho acústico das edificações.

Entende-se que a modelagem numérica pode contribuir para projetos acústicos mais eficientes. Os modelos numéricos necessitam - também - de dados de entrada precisos para a representação dos materiais e dos sistemas construtivos, o que só pode ser obtido a partir de ensaios em laboratórios criteriosos, dado que todo modelo numérico precisa de validação e calibração. Esse argumento valoriza sobremaneira o trabalho. 


\section{Referências}

ASSOCIAÇÃO BRASILEIRA DE NORMAS TÉCNICAS. NBR 14859: lajes pré-fabricadas de concreto: parte 1: vigotas, minipainéis e painéis: requisitos. Rio de Janeiro, 2016.

BET, K. et al. Avaliação do desempenho acústico de laje nervurada com diferentes composições de pisos. In: ENCONTRO NACIONAL DE CONFORTO NO AMBIENTE CONSTRUÍDO, João Pessoa, 2019. Anais [...] Porto Alegre: ANTAC, 2019.

BIES, D. A.; HANSEN, C. H. Engineering noise control: theory and practice. $4^{\text {th }}$ ed. Nova York: CRC Press, 2017.

BORGES, J. K. et al. An experimental study on the use of waste aggregate for acoustic attenuation: EVA and rice husk composites for impact noise reduction. Construction and Building Materials, v. 161, p. 501$508,2018$.

CANIATO, M. et al. Impact sound of timber floors in sustainable buildings. Building and Environment, v. 120, p. 110-22, 2017.

HASSAN, O. A. B. Building acoustics and vibration: theory and practice. London: World Scientific Publishing Company, 2009.

HOPKINS, C. Determination of vibration reduction indices using wave theory for junctions in heavyweight buildings. Acta Acustica united with Acustica, v. 100, n. 6, p. 1056-1066, 2014.

HOPKINS, C. Sound Insulation. $2^{\text {nd }}$ ed. Burlington: Elsevier, 2012.

HOPKINS, C; TURNER, P. Field measurement of airborne sound insulation between rooms with nondiffuse sound fields at low frequencies. Applied Acoustics, v. 66, n. 12, p. 1339-1382, dez. 2005.

INTERNATIONAL ORGANIZATION FOR STANDARDIZATION. ISO 10140-2: acoustics: laboratory measurement of sound insulation of building elements: part 2: measurement of airborne sound insulation. Genebra, 2010a.

INTERNATIONAL ORGANIZATION FOR STANDARDIZATION. ISO 10140-3: acoustics: laboratory measurement of sound insulation of building elements: part 3: measurement of impact sound insulation. Genebra, 2010b.

INTERNATIONAL ORGANIZATION FOR STANDARDIZATION. ISO 10140-4: acoustics: laboratory measurement of sound insulation of building elements: part 4: measurement procedures and requirements. Genebra, 2010c.

INTERNATIONAL ORGANIZATION FOR STANDARDIZATION. ISO 10140-5: acoustics: laboratory measurement of sound insulation of building elements: part 4: requirements for test facilities and equipment. Genebra, 2010d.

INTERNATIONAL ORGANIZATION FOR STANDARDIZATION. ISO 717-1: acoustics: rating of sound insulation in buildings and of buildings elements: part 1: airborne sound insulation. Genebra, $2013 \mathrm{a}$.

INTERNATIONAL ORGANIZATION FOR STANDARDIZATION. ISO 717-2: acoustics: rating of sound insulation in buildings and of building elements: part 2: impact sound insulation. Genebra, $2013 \mathrm{~b}$.

KIM, J. H.; JEON, J. Y. Effects of vibration characteristics on the walking discomfort of floating floors on concrete slabs. The Journal of the Acoustical Society of America, v. 136, n. 4, p. 1702, 2014.

LEE, W.; KIM, K.; LIM, S. Improvement of floor impact sound on modular housing for sustainable building. Renewable and Sustainable Energy Reviews, v. 29, p. 263-275, jan. 2014.

MOSHARROF, M. S. et al. An improved prediction model for the impact sound level of lightweight floors: introducing decoupled floor-ceiling and beam-plate moment. Acta Acustica United with Acustica, v. 97, n. 2, p. 254-65, 2011.

NUNES, M. F. O.; ZINI, A.; PAGNUSSAT, D. T. Desempenho acústico de sistemas de piso: estudos de caso para isolamento ao ruído aéreo e de impacto. Acústica e Vibrações, v. 46, 2014.

OLIVEIRA, M. F.; PATRICIO, J. V. Impact noise of non-homogeneous floors: analysis of different input parameters for computational modeling predictions. Journal of Civil Engineering and Architecture, v. 11, n. 3, p. 274-281, 2017. 
PARK, H. S.; YOON, D. Y.; CHO, T. Influence of plan configuration on low frequency vibroacoustic behaviour of floating floor with low natural frequency. Applied Acoustics, v. 158, 2020.

PATRÍCIO, J. V. Acústica nos edifícios. 7. ed. Lisboa: Verlag Dashöfer, 2018.

PATRÍCIO, J. V. Can Beam-block floors be considered homogeneous panels regarding impact sound insulation? Building Acoustics, v. 8, n. 3, p. 223-236, set. 2001.

SCHIAVI, A. Improvement of impact sound insulation: a constitutive model for floating floors. Applied Acoustics, v. 129, p. 64-71, 2018.

SCHIAVI, A.; TARIZZO, P.; ASTOLFI, A. Considerations on the impact sound pressure level of bare floor slabs in hollow brick and concrete. In: INTERNOISE, Lisboa, 2010. Anais [...] Lisboa: Institute of Noise Control Engineering, 2010.

SEMPRINI, G.; BARBARESI, L. Experimental results of flanking transmission in hollow brick and concrete-slab floor. In: EUROREGIO, Merano, 2013. Anais [...] Merano: Associazione Italiana di Acustica, 2013.

SEMPRINI, G. et al. acoustical behaviour of bare floor in hollow brick and concrete: Italian building technology. In: INTER-NOISE, Lisboa, 2010. Anais [...] Lisboa: Sociedade Portuguesa de Acústica, 2010.

TOMITAKA, R.; NOJIMA, R.; MASUDA, K. Study on reduction of floor impact sound by heavy impact source using double-layered ceiling with damping by granular materials. Journal of Environmental Engineering, v. 81, n. 719, p. 29-39, 2016.

YOO, S. Y.; JEON, J. Y. Investigation of the effects of different types of interlayers on floor impact sound insulation in box-frame reinforced concrete structures. Building and Environment, v. 76, p. 105-112, 2014.

\title{
Agradecimentos
}

Os autores agradecem a Construrohr pelo apoio e disponibilização dos resultados.

\author{
Maria Fernanda Oliveira \\ itt Performance | Unisinos | Rua Unisinos, 950, Cristo Rei | São Leopoldo RS - Brasil | CEP 93022-750 | Tel.: (51) 3590-8887 | E-mail: \\ mariaon@unisinos.br

\section{Rafael Ferreira Heissler} \\ itt Performance | Unisinos | E-mail: rheissler@unisinos.br \\ Felipe Schneider de Lima \\ Setor de Engenharia | Construrohr | Rodovia 122 km 22 | Bom Princípio - RS - Brasil | CEP 95765-000 | Tel.: (51) 99849-6453 | E-mail: \\ felipe.schneiderlima@outlook.com

\section{Fernanda Pacheco} \\ itt Performance | Unisinos | E-mail: fernandapacheco@unisinos.br \\ Bernardo Fonseca Tutikian \\ itt Performance | Unisinos | E-mail: bftutikian@unisinos.br
}

\author{
Ambiente Construído \\ Revista da Associação Nacional de Tecnologia do Ambiente Construído \\ Av. Osvaldo Aranha, $99-3^{\circ}$ andar, Centro \\ Porto Alegre - RS - Brasil \\ CEP 90035-190 \\ Telefone: +55 (51) 3308-4084 \\ www.seer.ufrgs.br/ambienteconstruido \\ www.scielo.br/ac \\ E-mail: ambienteconstruido@ufrgs.br
}

